\title{
Analyzing standard RF Waveform Designs for WPT
}

\author{
Sumit Gautam ${ }^{\dagger}$, Sumit Kumar ${ }^{\dagger}$, Symeon Chatzinotas, and Björn Ottersten \\ Interdisciplinary Centre for Security, Reliability and Trust (SnT), University of Luxembourg, Luxembourg. \\ Email: \{sumit.gautam,sumit.kumar,symeon.chatzinotas,bjorn.ottersten\}@uni.lu \\ ${ }^{\dagger}$ These authors have contributed equally to this work.
}

\begin{abstract}
The possibility to harvest energy from ambient radio-frequency $(\mathrm{RF})$ sources has intrigued humankind since past several decades. In this context, there has been a tremendously growing research interest in the field of wireless power transfer (WPT) using the RF regime of the electromagnetic (EM) spectrum. In this paper, we investigate the aspect of real-time energy harvesting (EH) via different types of waveform designs such as Orthogonal Frequency Division Multiplexing (OFDM), Square, Triangular, Sinusoidal, and Sawtooth. We make use of a Software Defined Radio (SDR) and a Powercast P21XXCSREVB EH module to carry out the experiments on practical device performance. Specifically, we are interested in obtaining some insights based on the comparison between the aforementioned waveform designs from the perspectives of separation distance between the USRP and P21XXCSR-EVB EH module, and power emission via USRP. Correspondingly, the demonstration of the EH is provided in terms of the above-mentioned investigation metrics, while seeking the best waveform to support WPT.

Index Terms-RF-Energy Harvesting, Universal Software Radio Peripheral (USRP) - Software Defined Radio (SDR), Wireless Power Transfer (WPT).
\end{abstract}

\section{INTRODUCTION}

With each passing year, we are inching closer-and-closer towards realizing a smart and connected society. In the process, it is important to note the increasing presence of low-power consuming internet-of-things (IoT) devices. These devices primarily serve the purpose of data collection in the form of various types of sensors (e.g., temperature, pressure, etc.), however, bounded by the strict battery limitations. To address the battery charging issues where the devices are placed at very remote places, while also taking into consideration the data exchange process; the possibility to use radio-frequency (RF) signals as a wireless charging means along with the traditional information transmission has gained due attention [1]-[4].

Recent research developments in the area of wireless power transfer (WPT) using RF signals have focused on laying important theoretical foundations pertaining to several key aspects in the domain, such as, waveform designing, rectifierbased modeling, beamforming algorithms, co-existence with traditional wireless communications systems, etc., [5]. While the theoretical findings motivates towards the use of multi-sine type waveform designs (e.g., Orthogonal Frequency Division Multiplexing (OFDM)) for WPT [5], recent experimental findings still back the adoption of traditional Sinusoidal waveform in the $915 \mathrm{MHz}$ operation regime [6]. Additionally, the Square waveform [7] and extended Square, i.e., Rectangular waveform [8] are also potential candidates to be considered as possible inputs for efficient WPT. In this context, seeking the best

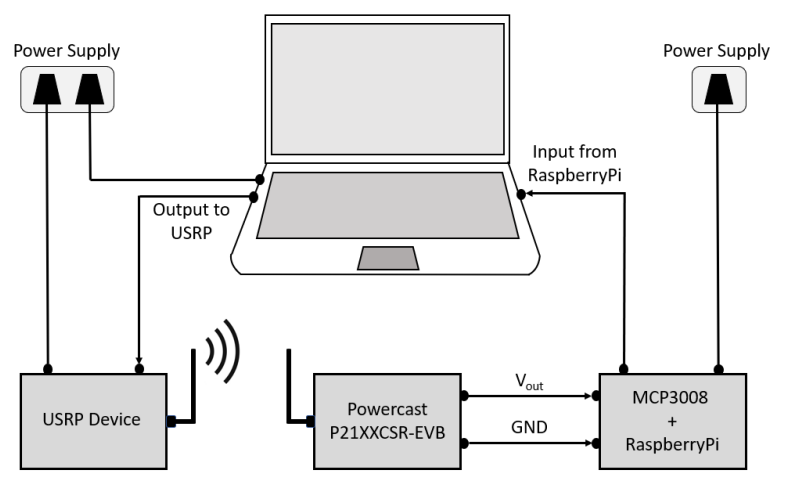

Fig. 1: System set-up comprised of a laptop, USRP, Powercast P21XXCSR-EVB energy harvester, MCP3008 and RaspberryPi.

suitable waveform design for WPT is important, which is the main focus of this work.

In this paper, we present a comparison between five types of traditional waveform designs based on real-time experimental values to bring more clarity on the best type of waveform design suitable for WPT mechanism. We perform an experimental analysis of the output voltage measurement from the Powercast P21XXCSR-EVB energy harvesting (EH) module, where the WPT process is facilitated by a Software Defined Radio (SDR). The SDR transmits the considered waveform designs over a wireless medium, which are individually utilized to test the efficacy of the WPT mechanism. Further sections of this paper are organized as follows. Section II provides an introduction to the system model comprising the hardware and software specifications. The details on real-time on-board evaluation and corresponding analysis is presented in Section III. Discussions on major outcomes are presented in IV, followed by concluding remarks in Section V.

\section{SyStem Set-uP - HARDWARE AND SOFTware}

In this section, we describe the considered system model in detail. Our experimental set-up is comprised of a laptop, Universal Software Radio Peripheral (USRP) B210, couple of $2.45 \mathrm{GHz}$ omni-directional antennas, Powercast P21XXCSREVB EH module, MCP3008, and a RaspberryPi. Specifically, the laptop provides instructions to the USRP pertaining to the chosen waveform and corresponding wireless emission. The USRP then emits a specific waveform signal over the wireless medium for the $\mathrm{EH}$ module to harness its energy. Since the output readings are in an analog form, we make use of MCP3008, which is a 10-bit Analog-to-Digital Converter 


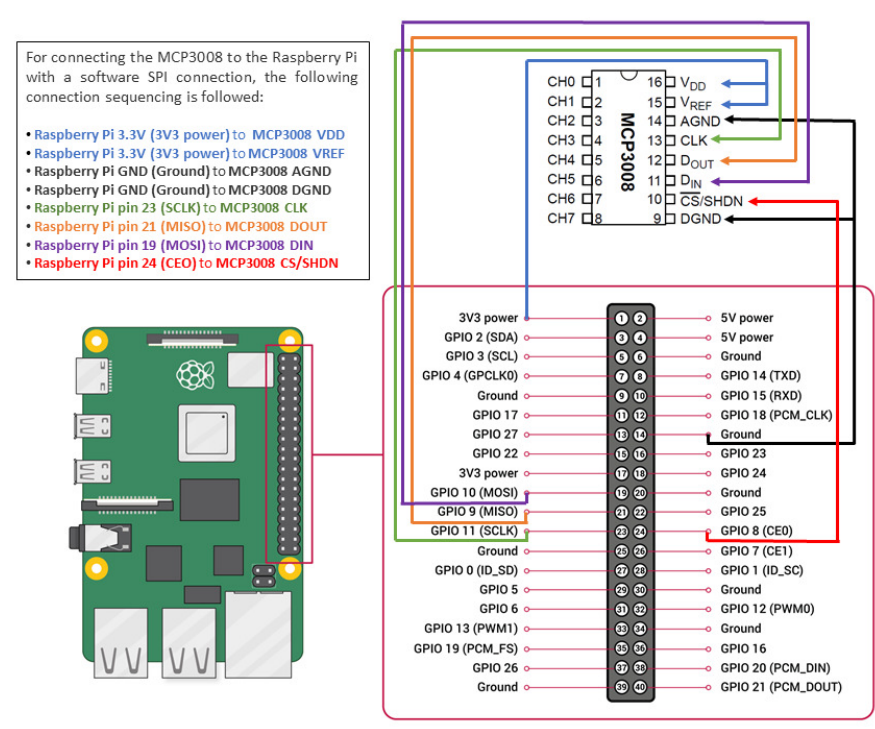

Fig. 2: Connection instructions between MCP3008 and RaspberryPi for interpreting $\mathrm{V}_{\text {out }}$ from the Powercast P21XXCSR-EVB.

(ADC). The ADC is then connected to a RaspberryPi, which logs the digital data results for interpretation. The schematics for overall system model and interconnection procedures between the MCP3008 and RaspberryPi are as shown in Fig. 1 and Fig. 2, respectively. A snapshot of the experimental setup is provided in Fig. 3. Further descriptions on the involved equipment are discussed below.

\section{A. USRP device and GNU Radio}

For our experiments, we make use of the Ettus USRP B210 SDR [9] hardware. It is a dual channel Field Programmable Gate Array (FPGA) based transceiver with a frequency span of $70 \mathrm{MHz}-6 \mathrm{GHz}$ and provides $56 \mathrm{MHz}$ of real time bandwidth. Using an SDR enables us to transmit a variety of waveforms using the same hardware. To transmit different waveforms, we use dual-band omni-directional antennas Vert2450 [10] which has frequency range of $2.4-2.5 \mathrm{GHz}$ and $4.9-5.9 \mathrm{GHz}$. On the software side, we used GNU Radio [11] which is used to program the USRP B210 SDR and design multiple waveforms. Under GNU Radio, waveforms are designed using a Graphical User Interface (GUI) based environment as shown in Fig. 4. We have used five different waveform designs: (i) Square, (ii) Triangle, (iii) Sinusoidal, (iv) Saw-tooth, at sampling rate of $20 \mathrm{MHz}$ and baseband frequency of $10 \mathrm{MHz}$, and (v) OFDM at

TABLE I: Equipment details related to the system set-up.

\begin{tabular}{|l|l|}
\hline Equipment & Details \\
\hline Laptop & $\begin{array}{l}\text { Dell Latitude 5490, 16GB RAM, Core i7, Ubuntu } \\
18.04, \text { GNU Radio Version 3.7 }\end{array}$ \\
\hline SDR & USRP B210: Ettus Research-National Instruments. \\
\hline Antennas & 2.45GHz, Omnidirectional, Gain: 3dBi \\
\hline RF-EH Module & Powercast P21XXCSR-EVB \\
\hline MCP3008 & 10-bit Analog-to-Digital Converter(ADC),150 Ksps \\
\hline RaspberryPi & Version 4, Python Ver-3 data from ADC \\
\hline Connectors & $\begin{array}{l}\text { Laptop to USRP: USB3.0, EH module to ADC } \\
\text { \& ADC to RaspberryPi: normal jumper cables, } \\
\text { RaspberryPi to Laptop: Ethernet Cat5. }\end{array}$ \\
\hline
\end{tabular}

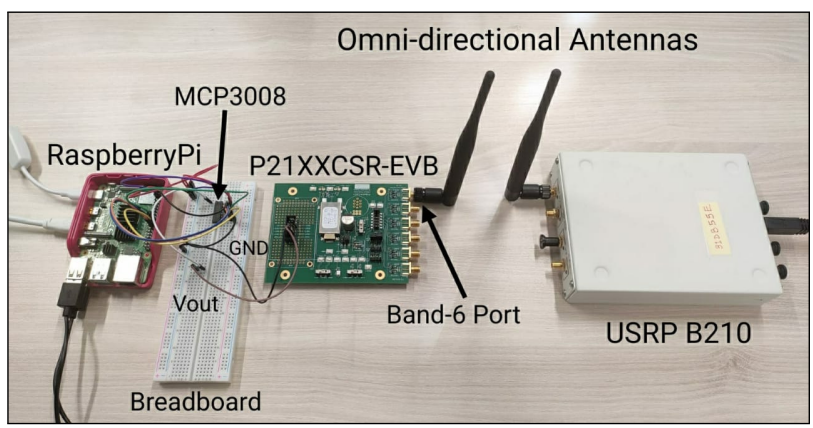

Fig. 3: Experimental set-up for analyzing WPT via various waveform designs transmitted over wireless medium using an SDR.

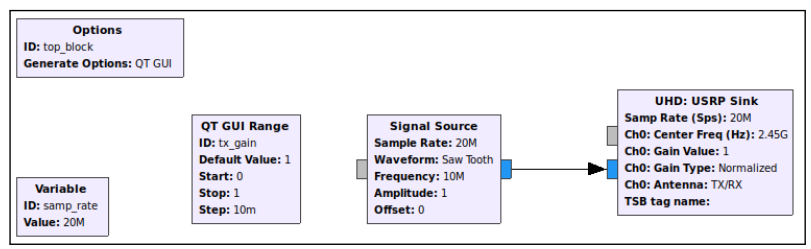

Fig. 4: GNU Radio GUI programming environment

sampling rate of $20 \mathrm{MHz}$ and 64 point FFT. For OFDM, we used gr-ieee802-11 package of GNU Radio which is capable of generating standard compliant IEEE 802.11g waveforms [12]. Analog gain of the waveforms is tuned on the fly using GNU Radio GUI during transmission.

\section{B. Powercast P21XXCSR-EVB EH Module}

The multi-band enabled Powercast P21XXCSR-EVB EH module provides six frequency band options to harvest energy from: GSM-850 uplink, Europe RFID \& GSM-850 downlink, ISM USA \& GSM-900 uplink, GSM-1800, GSM-1900 uplink, and Wi-Fi 2.4GHz. We use the Band 6 (Wi-Fi 2.4GHz), and choose Capacitor C6, with DEFAULT switch mode in S1. The switch S2 is kept in OFF mode while S3 is kept at MEAS/VCC. The default maximum output voltage i.e., $\mathrm{V}_{\text {out }}$ for this setting is 3.3 Volts [13], [14].

\section{C. $M C P 3008$ ADC}

In order to interpret the results from the $\mathrm{EH}$ module, i.e., $\mathrm{V}_{\text {out }}$, we need MCP3008 [15] to convert the received analog output into digital form. The 10-bit ADC provides a maximum value of 1023 in our experiment, which corresponds to 3.3 Volts. The $\mathrm{V}_{\text {out }}$ from $\mathrm{EH}$ module may be connected to any of the channels $(\mathrm{CH} 0-\mathrm{CH} 7)$ of MCP3008. For our experiment, we use the $\mathrm{CH} 0$ to interpret the results via RaspberryPi.

TABLE II: Software/Parameter stipulations for experiments.

\begin{tabular}{|l|l|}
\hline Feature & Specifications \\
\hline Frequency Band & $802.11 \mathrm{~g}-2.4 \mathrm{GHz}$ \\
\hline Carrier Frequency & $2.45 \mathrm{GHz}$ \\
\hline Sampling Rate & $20 \mathrm{MHz}$ \\
\hline Baseband Frequency & $10 \mathrm{MHz}$ \\
\hline OFDM Details & $\begin{array}{l}64(52 \text { sub-carriers utilized, } 11 \text { Guard-band \& } \\
1 \mathrm{DC}, \text { BPSK) }\end{array}$ \\
\hline Sample tapping rate & 100 samples collected per second \\
\hline RF-EH Settings & $\begin{array}{l}\text { Band 6, Capacitor }: \text { C6, S1 : DEFAULT, } \\
\text { S2 : OFF, S3 : MEAS/VCC }\end{array}$ \\
\hline
\end{tabular}




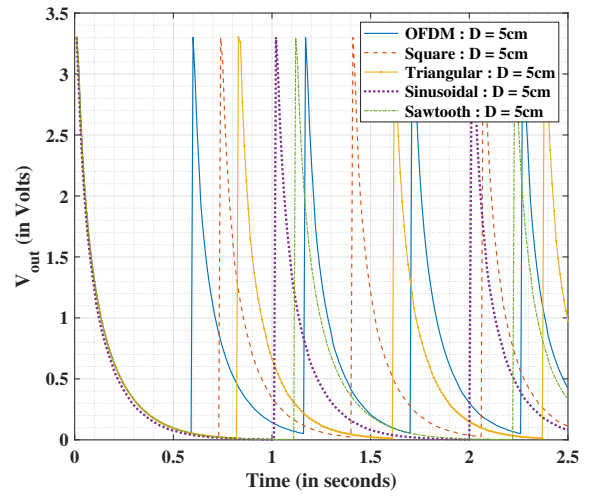

(a)

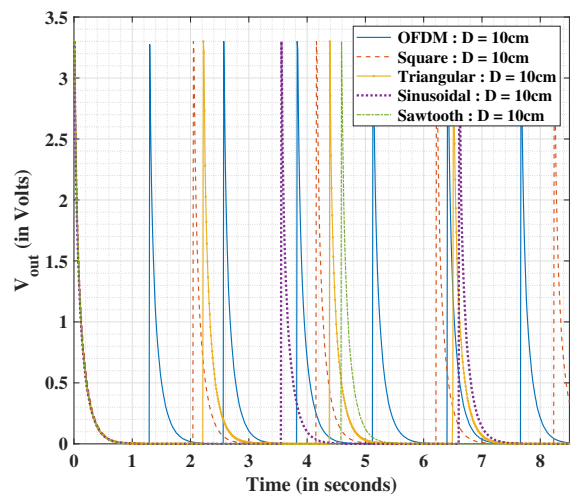

(b)

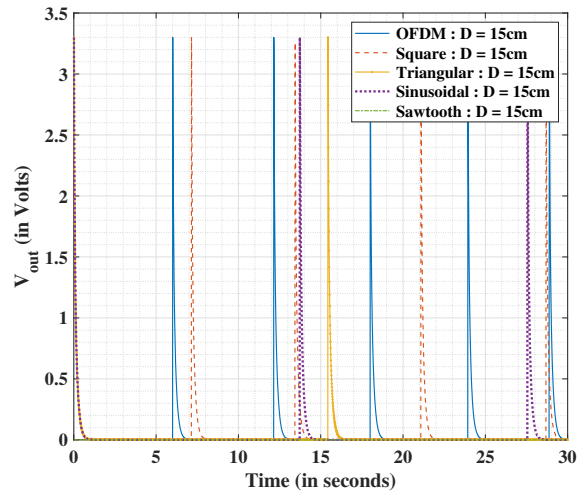

(c)

Fig. 5: Comparison between the peak-to-peak $\mathrm{V}_{\text {out }}$ obtained via considered waveform designs: OFDM, Square, Triangular, Sinusoidal, and Sawtooth, for a separation distance between the USRP and P21XXCSR-EVB EH module (a) D = 5cm, (b) D = 10cm, and (c) D = 15cm.

\section{Logging the data via RaspberryPi}

We use the RaspberryPi v4 [16] to log the voltage values produced by the considered EH device. MCP3008 is interfaced to the RaspberryPi and digitized voltage values from MCP3008 are obtained through the Serial Programming Interface (SPI). We use Python to prepare the data-logging program and setting the sampling rate of MCP3008. For our experiments, we assume a sampling rate of 100 samples per second (sps), which is sufficient for our purpose.

More details on the equipment and parameter selections for software configuration are provided in Table I and Table II, respectively. In the succeeding section, we discuss the considered experimental findings and corresponding analysis.

\section{REAL-TIME ON-BOARD Evaluation AND ANALysis}

For the hardware experiments, we use the Industrial Scientific and Medical (ISM) band of $2.4 \mathrm{GHz}$, where the central frequency of $2.45 \mathrm{GHz}$ is considered. The waveform designs are sampled at $20 \mathrm{MHz}$ and the corresponding instructions are sent via laptop to the USRP using the GNU Radio environment. The USRP emits the corresponding signal with specific waveform to the Powercast P21XXCSR-EVB EH module over the wireless medium. The USRP and the EH module are separated by a small margin. This is due to the fact that the USRPs for institutional use are limited by extremely small maximum transmit power. The calibrated values for each waveform corresponding to $100 \%$ of the peak transmitted power by the USRP are measured as follows: OFDM (-23 $\mathrm{dBm})$, Square $(-19 \mathrm{dBm})$, Triangular $(-18 \mathrm{dBm})$, Sinusoidal $(-14.75 \mathrm{dBm})$, and Sawtooth $(-18.4 \mathrm{dBm})$.

The experiments are performed in real-time on the specified system set-up. We evaluate the effects of increasing the distance and transmit power on the received $\mathrm{V}_{\text {out }}$ from the $\mathrm{EH}$ module. These values are logged with the help of MCP3008 $\mathrm{ADC}$ and RaspberryPi, as explained before. Specifically, the interpretation of results are primarily dependent on the charging and discharging cycles of the capacitor present in the EH module. In this context, it is noteworthy that the peakto-peak voltage values will differ according to the type of waveform chosen at the transmit node, i.e., USRP. From a real-time perspective, it is difficult to start the experiment from one peak itself. Hence, we start the collection of samples at any random time after the USRP starts the transmission process. In the process, we collect 100 samples per second with a maximum of 5000 sample collections. After logging the outcomes, we align the first peaks corresponding to the various waveform designs used considered for the experiments. This would facilitate in better interpretation of the results. In the following, we present the results and discussions related to the WPT based on variation of distance between the USRP and EH module, and the transmit power metric at the USRP.

\section{A. Impact on WPT by varying the separation distance between the USRP and Powercast P21XXCSR-EVB EH module}

From an intuitive view-point, it is easy to interpret the effect of increasing the separation distance between the power transmitter and EH module, on WPT. Concretely, the analysis of RF-enabled wireless communications systems provides better understanding on the fate of wireless signal with specific waveform and power. Due to the environmental penetration losses, the overall received wireless signal becomes weak, which gets weaker with the increasing separation distance between the transmit source and receiver. This is well explained by the Friis transmission formula for the free space path loss [17]

$$
\frac{\mathrm{P}_{\mathrm{T}}}{\mathrm{P}_{\mathrm{R}}} \propto \frac{1}{\mathrm{D}^{2}}
$$

where $\mathrm{P}_{\mathrm{T}}$ is the transmit power, $\mathrm{P}_{\mathrm{R}}$ is the received power at the receiver and $\mathrm{D}$ is the separation distance between the transmit antenna and receive antenna. In our experiment, $\mathrm{P}_{\mathrm{T}}$ is the transmit power from the USRP (kept at 100\%), $P_{R}$ is the received power at the $\mathrm{EH}$ module and $\mathrm{D}$ is the separation distance between the antennas of the USRP and EH module.

In Fig. 5, we show the effect of increasing the distance on the harvested energy via various considered waveforms, viz-aviz., OFDM, Square, Triangular, Sinusoidal, and Sawtooth. We observe that the separations between the peak-to-peak voltages obtained at the receiver via each corresponding waveform 


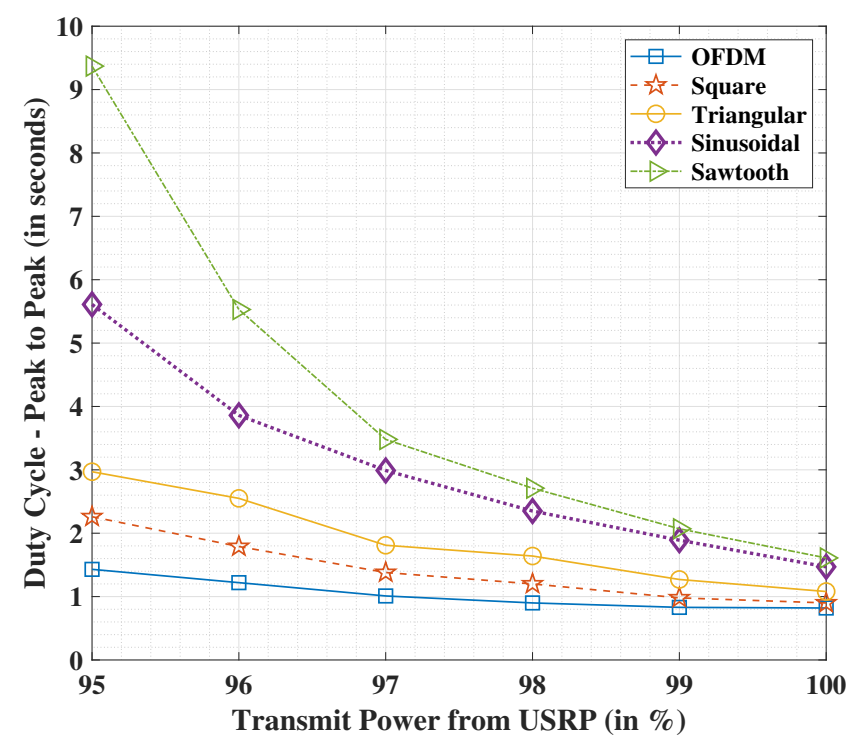

Fig. 6: Effect of varying transmit power from the USRP on the Duty Cycle - Peak to Peak for the considered waveform designs.

continues to grow with increasing separation distances between the antennas of the USRP and EH module. ${ }^{1}$ Further, it is noted that the peaks appear less frequently with increasing separation distance, indicating that the capacitor requires higher time to charge. In terms of the waveform designs, we find that OFDM is the best suitable multi-carrier candidate among all the chosen waveform designs for comparison, while the Square waveform is found to be the best suitable single carrier candidate for facilitating the $\mathrm{EH}$ process in all the cases.

\section{B. Impact on WPT with varying the transmit power of USRP}

It is a well-known fact that an increase or decrease in the transmit power causes same corresponding effect at the receive node. This implies that the received power increases with growing transmit power values and vice-versa, while the pathloss phenomenon mentioned in (1) also holds. In this context, we perform our experiments by keeping a fixed separation distance between the antennas of the USRP and EH module with $\mathrm{D}=7.5 \mathrm{~cm}$, while changing the transmit power values at the USRP device. In the process, we take into consideration the sample differences (SD) between the first peak and second peak of $\mathrm{V}_{\text {out }}$ for each of the considered waveform designs. This indicates the charging-discharging cycles of the capacitor present in the EH module, which we term as the Duty Cycle - Peak to Peak calculated as SD per 100 received sps.

We report in Fig. 6 the effect of varying transmit power from the USRP on the Duty Cycle - Peak to Peak for the considered waveform designs. In-line with the abovementioned intuitive discussion, we find that the Duty Cycle for the capacitor decreases with the increase in the transmit power

\footnotetext{
${ }^{1}$ Note that short separation distances are considered for investigation since power amplifiers are not used during the transmission process from USRP.
}

from the USRP. This also indicates a faster charging process and the waveform with the least Duty Cycle at any value of transmit power would be considered most suitable for the EH mechanism. We note that in this case, OFDM outperforms the other considered waveform designs from the multi-carrier perspective, while Square wave is found to be most suitable candidate for the $\mathrm{EH}$ process from a single carrier view-point.

\section{Major Outcomes AND InTERPRETIVE Discussion}

Based on our experiments, we find that the OFDM waveform (multi-sine) is found to be the best suitable candidate among all the considered waveform designs, viz., OFDM, Square, Triangular, Sinusoidal, and Sawtooth. However, the Square waveform also provides an appreciable performance in the single carrier waveform category. The efficiency of the multi-carrier OFDM waveform is justified by the fact that the EH module sees the multi-sine waves as the supplier of frequent peak voltages, which improves the charging process at the capacitor placed in the EH module. Surprizingly, the Triangular wave is found to provide better performances in comparison to the Sinusoidal wave for several investigative instances. In contrast, the Sawtooth wave performs the worst due to obvious reasons of not being able to provide frequent peak-to-peak voltage values. Since multi-carrier systems with high number of sub-carriers may resemble a continuous highfrequency based Square wave, therefore it is needless to mention that such waveform designs which provides highly frequent peak-to-peak voltage values are to be considered the best for EH mechanism. As an interesting trade-off, this phenomenon appears to be the opposite of what we want for a normal information transmitter to avoid non-linear distortion.

\section{CONCLUSion}

In this paper, we demonstrated the real-time analysis of WPT via various waveform designs viz., OFDM, Square, Triangular, Sinusoidal, and Sawtooth, transmitted over a wireless medium using an SDR. For the experimental tractability and for interpreting the $\mathrm{V}_{\text {out }}$ from the Powercast P21XXCSR-EVB EH module, we presented a mechanism to convert the received analog data to a digital interpretative format using MCP3008 $\mathrm{ADC}$ and RaspberryPi. Our results highlight that OFDM is the best suitable multi-carrier candidate among the considered set of waveform designs, while Square waveform is found to be ideal single carrier waveform for facilitating the EH process.

\section{REFERENCES}

[1] L. R. Varshney, "Transporting information and energy simultaneously," in 2008 IEEE Int. Symp. on Information Theory, 2008, pp. 1612-1616.

[2] S. Gautam, E. Lagunas, S. Chatzinotas, and B. Ottersten, "Relay Selection and Resource Allocation for SWIPT in Multi-User OFDMA Systems," IEEE Trans. Wireless Commun., vol. 18, no. 5, pp. 2493-2508, 2019

[3] S. Gautam, T. X. Vu, S. Chatzinotas, and B. Ottersten, "Cache-Aided Simultaneous Wireless Information and Power Transfer (SWIPT) With Relay Selection," IEEE J. Sel. Areas Commun., vol. 37, no. 1, pp. 187201, Jan 2019.

[4] S. Gautam and et al., "Multigroup Multicast Precoding for Energy Optimization in SWIPT Systems With Heterogeneous Users," IEEE Open Journal of the Communications Society, vol. 1, pp. 92-108, 2020. 
[5] B. Clerckx and et al., "Fundamentals of Wireless Information and Power Transfer: From RF Energy Harvester Models to Signal and System Designs," IEEE J. Sel. Areas Commun., vol. 37, no. 1, pp. 4-33, 2019.

[6] N. Ayir, M. F. Trujillo Fierro, T. Riihonen, and M. Allén, "Experimenting Waveforms and Efficiency in RF Power Transfer," in 2019 IEEE MTT-S International Microwave Symposium (IMS), 2019, pp. 1140-1143.

[7] K. Yamaguchi, T. Hirata, and I. Hodaka, "Using Square Wave Input for Wireless Power Transfer," International Journal of Electrical \& Computer Engineering (2088-8708), vol. 6, no. 1, 2016.

[8] N. Shanin, L. Cottatellucci, and R. Schober, "Markov Decision Process Based Design of SWIPT Systems: Non-Linear EH Circuits, Memory, and Impedance Mismatch," IEEE Trans. Commun., vol. 69, no. 2, pp. 1259-1274, 2021.

[9] "Ettus Research," https://www.ettus.com/all-products/ub210-kit/, Accessed: 2021-02-28.

[10] "Vert-2450," https://www.ettus.com/all-products/vert2450/, Accessed:
2021-02-28.

[11] "GNU Radio," https://www.gnuradio.org/, Accessed: 2021-02-28.

[12] B. Bloessl, M. Segata, C. Sommer, and F. Dressler, "An IEEE 802.11 a/g/p OFDM Receiver for GNU Radio," in Proceedings of the second workshop on Software radio implementation forum, 2013, pp. 9-16.

[13] Powercast Corporation, P21XXCSR-EVB, https://www.powercastco. com/documentation/p21xxcsr-evb-datasheet/.

[14] H. Aboueidah and et al., "Characterization of RF energy harvesting at $2.4 \mathrm{GHz}$," in 2017 24th IEEE International Conference on Electronics, Circuits and Systems (ICECS), 2017, pp. 446-449.

[15] "MCP3008 ADC," https://cdn-shop.adafruit.com/datasheets/MCP3008. pdf, Accessed: 2021-02-28.

[16] "RaspberryPi-v4," https://www.raspberrypi.org/products/ raspberry-pi-4-model-b/, Accessed: 2021-02-28.

[17] H. T. Friis, "A note on a simple transmission formula," Proceedings of the IRE, vol. 34, no. 5, pp. 254-256, 1946. 